Theory of masers and maser sources 


\title{
Maser Theory: Old Problems and New Insights
}

\author{
M. D. Gray \\ Jodrell Bank Centre for Astrophysics, School of Physics and Astronomy, \\ Alan Turing Building, University of Manchester, \\ M13 9PL, Manchester, UK \\ email: Malcolm.Gray@manchester.ac.uk
}

\begin{abstract}
Maser theory continues to be driven by advances in observational techniques. Here, I consider the responses to VLBI with space-Earth baselines and cross-correlation spectroscopy (a re-consideration of coherence properties), routine observation in full-Stokes polarization (a re-casting of the polarization transfer equations), and long-term variability monitoring (3-D modelling of irregular domains).
\end{abstract}

Keywords. masers, molecular processes, radiative transfer, ISM: molecules, radio lines: general

\section{Introduction}

I consider in detail three areas of active maser research that may benefit from some new, or re-discovered, theoretical insight in addition to the available observational data. These areas are the coherence properties of astrophysical masers, the modelling of maser polarization, and the analysis of maser variability with 3 -D radiative transfer and saturation models.

\section{Coherence Properties}

Two types of coherence are discussed in Elitzur (1992): phase coherence and bandwidth coherence. Phase coherence is maintained between two rays from some point on a source, diverging through some beam angle $\theta$, and propagated over a distance $L$, provided that the phase difference $\Delta \phi=L \pi \theta^{2} / \lambda$ is much smaller than 1 radian. Large values of $L$, of order $10^{12} \mathrm{~m}$ in astrophysical masers, drove the conclusion that astrophysical masers are not phase coherent. This probably remains a valid conclusion, but the recent detection, with an interferometer including the RadioAstron instrument, of a $22-\mathrm{GHz}(\lambda=0.0136 \mathrm{~m}) \mathrm{H}_{2} \mathrm{O}$ maser in Cep A that is unresolved at an angular scale of $10 \mu \mathrm{as}$, improves the condition by several orders of magnitude. At the distance of Cep A, this angular scale corresponds to a linear distance of $\sim 10^{9} \mathrm{~m}$. Using this distance as $L$, but keeping the original estimate of $\pi \theta^{2}=3.14 \times 10^{-4}$, we obtain $\Delta \phi=2.42 \times 10^{6}$, so the conclusion of no phase coherence still appears safe, even with the smallest maser features so far discovered.

The second type of coherence discussed in Elitzur (1992) is bandwidth coherence. For a source to be bandwidth coherent, the essential requirement is that the homogeneous response profile of the transition is broader than the inhomogeneous profile. The homogeneous profile broadens as a maser saturates due to power, or saturation, broadening. This arises from Stark effect distortion of the molecule by the electric field of the maser radiation. According to Elitzur (1992), power broadening becomes sufficient to enforce bandwidth coherence for a brightness temperature of $T_{b}>3 \times 10^{14}(4 \pi / \Omega) \mathrm{K}$ for the 


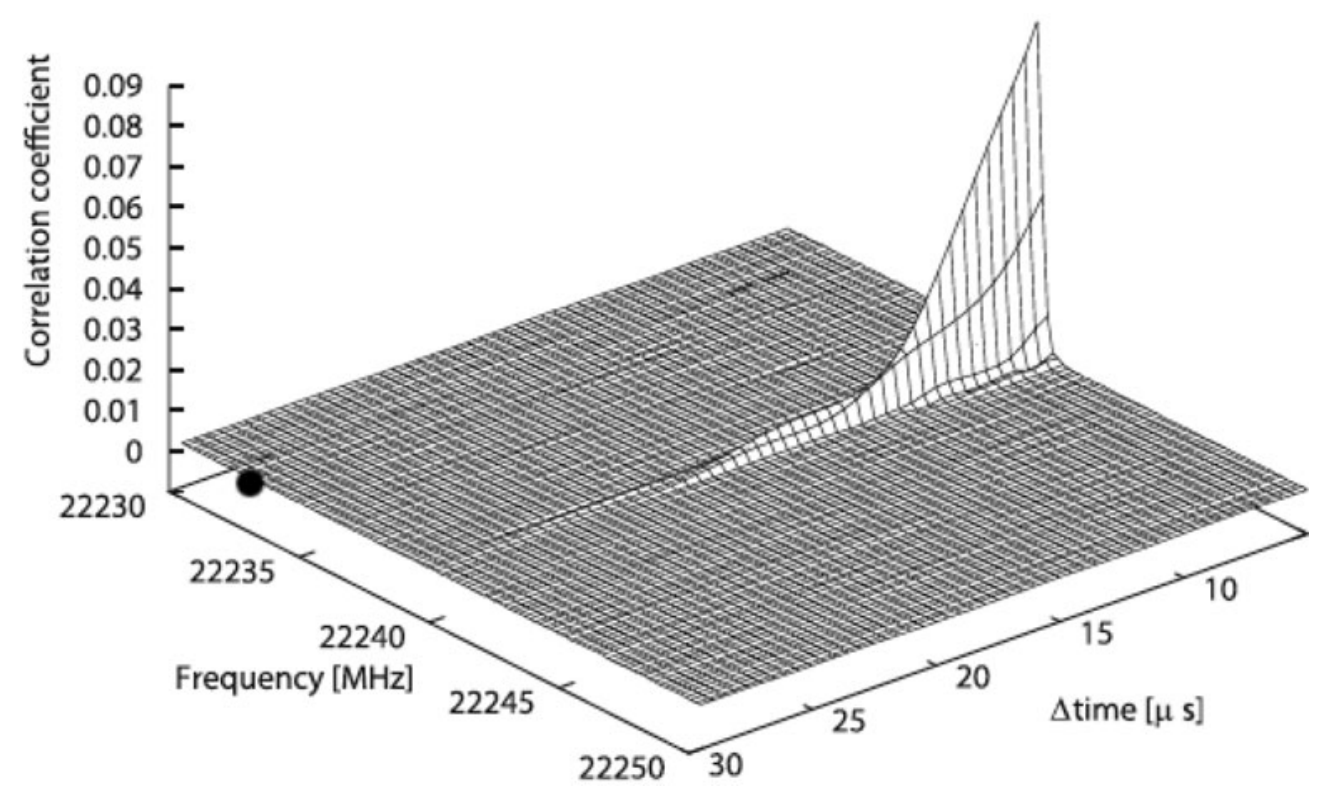

Figure 1. The coherence function as a function of time delay and of frequency across the line in an observation of the star-forming region $\mathrm{W} 3\left(\mathrm{H}_{2} \mathrm{O}\right)$ by Takefugi et al. (2016)

22- $\mathrm{GHz}$ transition of $\mathrm{H}_{2} \mathrm{O}$. For reasonable maser beam angles, see above, the brightest maser sources, particularly flares, could be marginally bandwidth coherent. For example water-maser flares have been observed in Orion with $T_{b}>10^{16} \mathrm{~K}$ (Kobayashi et al. 2000), and with $T_{b} \simeq 8 \times 10^{17} \mathrm{~K}$ (Strelnitskii 1982).

Recent evidence from observationally measured coherence functions (Takefugi et al. 2016) strongly suggests that very bright astrophysical masers are indeed bandwidth coherent. The key point is the shape of the decay of the coherence function (Fig. 1), which has a long tail towards longer time delays that is inconsistent with the Gaussian behaviour expected if the function were controlled by the inhomogeneous profile. Note that the relation between the coherence function and the line shape is a Fourier transform: a Gaussian inhomogeneous profile transforms to a Gaussian decay in the coherence function; a Lorentzian homogeneous profile transforms to an exponential, with a much slower decay. The observed coherence time in this example is $18 \mu \mathrm{s}$, suggesting a homogeneous width of $56 \mathrm{kHz}$. This compares to an observed inhomogeneous line profile width (full width at half power) of $96 \mathrm{kHz}$.

The Stark broadening effect by the electric field of the maser radiation imposes an ultimate limit on the brightness temperature, above which the line will be split into a distinctive pattern (Slysh 2003). The fact that a pattern of this type has not been observed suggests that maser beam angles for the brightest sources are of order $10^{-3}$, somewhat smaller than the estimates in Elitzur (1992). The measured coherence times in Takefugi et al. (2016) indicate that the brightness temperatures of some $\mathrm{H}_{2} \mathrm{O}$ maser features in $\mathrm{W} 3\left(\mathrm{H}_{2} \mathrm{O}\right)$ and $\mathrm{W} 49$ reach $8.5 \times 10^{18}$ and $6.2 \times 10^{22} \mathrm{~K}$ respectively.

Another old, but excellent, reference that covers many aspects of Doppler-broadened maser propagation, including the statistics of the observed radiation, which is related to the work described above, is Menegozzi \& Lamb (1978). 


\section{Polarization}

Perhaps no aspect of theoretical maser research has been more controversial. In particular, the case of Zeeman splittings small compared to the inhomogeneous line width has historically been very problematic. Another major source of difficulty is the use of different conventions to describe left- and right-handed polarization, and in the definitions of the Stokes parameters, particularly Stokes- $V$. Definitions conforming to the IEEE definition of right- and left-handed polarization and the IAU convention for Stokes $V$ are set out in Green et al. (2014).

One of the reasons that the polarization problem is so difficult is that four, coupled, simultaneous radiative transfer equations must be solved to recover the Stokes parameters. A piece of old insight into this problem was provided by Landi Degl'Innocenti (1987), who developed a method of writing the Stokes problem in the style of the integrating factor method that is used to write a formal solution of the scalar radiative transfer equation. The result is a Stokes vector solution that may be frequency and/or angle averaged as necessary, allowing the polarization problem to be cast into a Milne-Schwarzschild integral equation form, see, for example, King \& Florance (1964), by eliminating the radiation averages from the density matrix (DM) equations.

In detail, Landi Degl'Innocenti solves the full polarization radiative transfer equation, $d \mathbf{i} / d \tau=\eta \mathbf{i}$, in this case assuming negligible spontaneous emission, as $\mathbf{i}(\tau)=\mathrm{O}\left(\tau, \tau^{\prime}\right) \mathbf{i}\left(\tau^{\prime}\right)$, where $\tau$ is the optical depth and $\mathrm{O}\left(\tau, \tau^{\prime}\right)$ is a $4 \times 4$ matrix operator, the propagation operator that relates the dimensionless Stokes vector, $\mathbf{i}(\tau)$, at two different depths. In the limit where $\tau^{\prime} \rightarrow 0$, this operator multiplies the Stokes vector for a ray just entering the maser medium, and can be shown to be equal to (Landi Degl'Innocenti 1987),

$$
\mathrm{O}\left(\tau, \tau^{\prime}\right)=\mathrm{I}+\sum_{n=1}^{\infty} \frac{1}{n !} \int_{\tau^{\prime}}^{\tau} d \tau_{1} \int_{\tau^{\prime}}^{\tau} d \tau_{2} \ldots \int_{\tau^{\prime}}^{\tau} d \tau_{n} P\left\{\eta\left(\tau_{1}\right) \eta\left(\tau_{2}\right) \ldots \eta\left(\tau_{n}\right)\right\}
$$

In eq. 3.1, I is the identity matrix, and $P$ is an operator that orders the Stokes matrices, $\eta$ by depth, with the greatest depth on the left. A general Stokes matrix has seven unique elements, and may be written,

$$
\eta=\left[\begin{array}{cccc}
\eta_{i} & \eta_{q} & \eta_{u} & \eta_{v} \\
\eta_{q} & \eta_{i} & \rho_{v} & -\rho_{u} \\
\eta_{u} & -\rho_{v} & \eta_{i} & \rho_{q} \\
\eta_{v} & \rho_{u} & \rho_{q} & \eta_{i}
\end{array}\right]
$$

where many models assume $\eta_{u}=\rho_{v}=\rho_{q}=\rho_{u}=0$. The remaining terms, $\eta_{i}, \eta_{q}$ and $\eta_{v}$ are functions of the molecular populations or inversions and trigonometrical functions of the angle between the magnetic field, at a certain depth, and the ray direction, possibly with an additional continuum component.

Radiation averages derived from eq. 3.1 can now be used to eliminate these quantities from the DM equations. Such averages, denoted by an over-bar may be grouped into the convenient functions,

$$
\begin{aligned}
f^{0, \pm} & =\frac{1}{4 \pi} \oint 2\left(\bar{i}^{0, \pm}-\bar{q}^{0, \pm}\right) \sin ^{2} \theta d \Omega \\
h^{0, \pm} & =\frac{1}{4 \pi} \oint 2 \bar{v}^{0, \pm} \cos \theta d \Omega \\
g^{0, \pm} & =\frac{1}{4 \pi} \oint\left[\left(1+\cos ^{2} \theta\right) \bar{i}^{0, \pm}+\bar{q}^{0, \pm} \sin ^{2} \theta\right] d \Omega
\end{aligned}
$$


where the superscripts denote the transition type of the line shape function that has been used for the frequency integration. The DM equations that the results from eq. 3.3 need to be inserted into are somewhat complicated, but for a 3D model under the complete redistribution approximation, they are

$$
\begin{aligned}
\Delta^{+} & =\frac{\alpha^{+}}{D}\left\{\left(1+2 f^{0}\right)\left[1-\frac{\alpha^{-}}{\alpha^{+}}\left(g^{+}+h^{+}\right)+2\left(g^{-}+h^{-}\right)\right]+\left(\frac{\alpha^{-}}{\alpha^{+}} f^{+}-f^{-}\right)\left(g^{0}+h^{0}\right)\right. \\
& \left.+\frac{\alpha^{0}}{\alpha^{+}}\left[f^{-}\left(g^{+}+h^{+}\right)-f^{+}\left(1+2 g^{-}+2 h^{-}\right)\right]\right\}
\end{aligned}
$$

where $\Delta^{+}$is the inversion in the $\sigma^{+}$transition of a $J=1-0$ Zeeman group at a specific node or depth of the model. The $\alpha^{ \pm, 0}$ constants are the ratio of the pump and loss rates for the transition denoted by the superscript. The expression for $\Delta^{-}$has a similar structure to eq. 3.4, whilst the $\pi$-inversion is given by

$$
\begin{aligned}
\Delta^{0} & =\frac{\alpha^{0}}{D}\left\{1+2\left(g^{-}+h^{-}\right)+2\left(g^{+}-h^{+}\right)+3\left(g^{+} g^{-}-h^{+} h^{-}\right)+5\left(g^{+} h^{-}-g^{-} h^{+}\right)\right. \\
& -\frac{\alpha^{+}}{\alpha^{0}}\left[g^{0}\left(1+g^{-}+3 h^{-}\right)-h^{0}\left(1+3 g^{-}+h^{-}\right)\right] \\
& \left.-\frac{\alpha^{-}}{\alpha^{0}}\left[g^{0}\left(1+g^{+}-3 h^{+}\right)-h^{0}\left(1+3 g^{-}+h^{-}\right)\right]\right\} .
\end{aligned}
$$

The denominator, $D$, common to eq. 3.4 and eq. 3.5 is

$$
\begin{aligned}
D & =\left(1+2 f^{0}\right)\left[1+2\left(g^{-}+h^{-}\right)+2\left(g+-h^{+}\right)+3\left(g^{+} g^{-}-h^{+} h^{-}\right)+5\left(g^{+} h^{-}-g^{-} h^{+}\right)\right] \\
& +f^{+}\left[h^{0}\left(1+h^{-}+3 g^{-}\right)-g^{0}\left(1+g^{-}+3 h^{-}\right)\right] \\
& -f^{-}\left[h^{0}\left(1-h^{+}+3 g^{+}\right)+g^{0}\left(1+g^{+}-3 h^{+}\right)\right] .
\end{aligned}
$$

These equations now form a set of non-linear algebraic equations in the $\Delta^{ \pm, 0}$ at all positions or depths of the model.

\section{Nature of Masing Objects}

When the physical objects that support astrophysical masers are discussed, we often talk of them as 'clouds', but our knowledge of what they actually are is rather limited. An exception is a group of masers found in our own Solar System, known to originate from the comae of comets. The fact that we can reliably use masers for trigonometric parallax distance measurement strongly suggests that at least the masing objects are more like 'bullet' clouds than a transient wave-like phenomenon. Clues to the nature of maser objects include the geometry and frequency dependence of their emission, and likely also their variability, since the brightness of maser rays is strongly dependent on the available gain length in the line-of-sight. The considerations above call for a fully 3-D maser model with irregular geometry.

A good way to construct a general 3-D maser model is to use the finite-element method of discretization. Elements can in turn be generated from the Delaunay triangulation of an irregular distribution of points (nodes), see for example Zienkiewicz \& Taylor (2000). A triangulated domain of this type is shown in Fig. 2. The radiation solution for all nodes of the domain is carried out via a finite-element implementation of the CEP method (Elitzur \& Asensio Ramos 2006,Gray 2012) under a quasi-two-level CVR approximation at present. The solution for a certain optical thickness is straightforwardly used as a starting guess for the next, thicker, model, allowing highly saturated solutions to be 


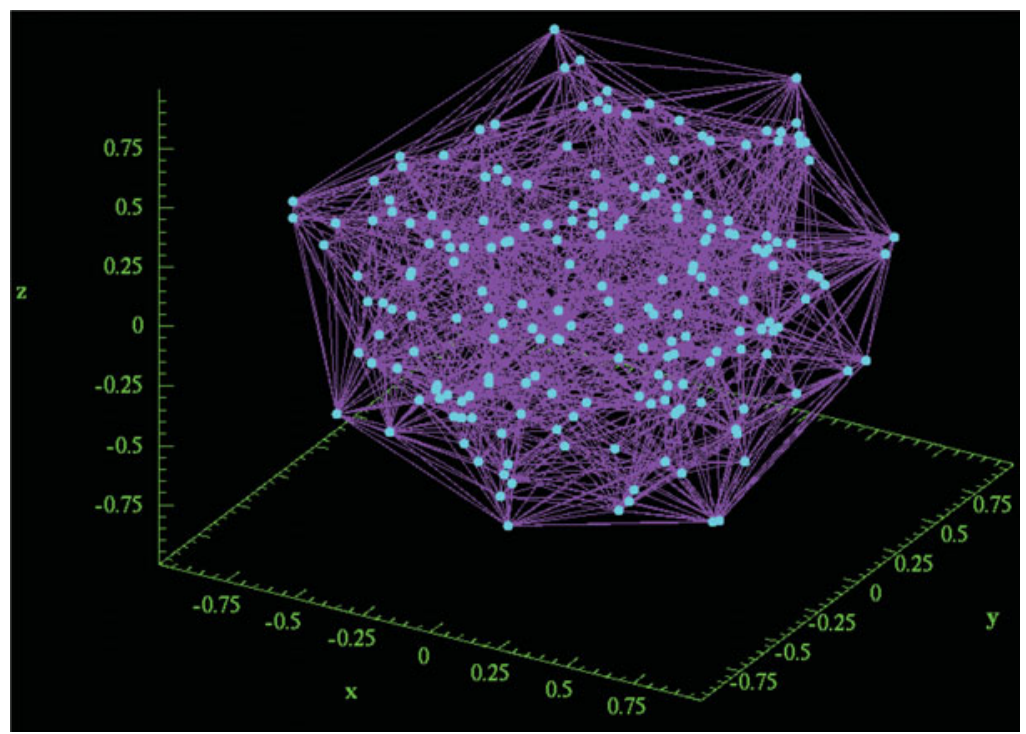

Figure 2. A triangulated domain of 1177 tetrahedral (simplex) elements with 202 nodes

obtained. If the model has $J$ nodes and $Q$ ray paths, then the inversion $\Delta_{i}$, at the $i$ th node, is given by

$$
\Delta_{i}-\left[1+\frac{i_{B G}}{4 \pi} \sum_{q=1}^{Q} \frac{a_{q}}{s_{q}^{2}} \sum_{n=0}^{\infty} \frac{1}{n !(n+1)^{1 / 2}}\left(\sum_{j=1}^{J} \Phi_{j, q} \Delta_{j}\right)^{n}\right]^{-1}=0
$$

where the functions $\Phi_{j, q}$ are constructed from the shape-functions of the elements of the nodes $j$ found along ray path $q$ to the target (node $i$ ). Solutions to eq. 4.1 show the expected concentration of the most saturated nodes to the outside of the model, and the least saturated nodes towards the centre. The $q$ th ray path has a facet area $a_{q}$ at a distance $s_{q}$ from the target.

Once nodal solutions have been computed, formal solutions can be calculated for the brightnesses of rays along lines of sight to an observer's position. These can be converted to maps that show the expected decrease in the observed size of the cloud with increasing amplification and saturation, for example Alcock \& Ross (1985).

It is possible to investigate maser variability by simulating rotation of the cloud depicted in Fig. 2. The simulation was performed by computing the radiation transfer solution in the frame of the cloud, which can have no internal velocity gradients assuming that it rotates as a solid body. An external observer then sees the cloud with a Doppler correction that appears mostly as broadening with a small shift in the peak frequency of the maser line. The plane perpendicular to the rotation axis was chosen so as to present the longest axis of the domain periodically to the observer.

Variability of Class 2 methanol masers has been investigated in detail by Goedhart, Gaylard \& van der Walt (2004), where light curves of several years duration are plotted for a variety of sources that show patterns that include monotonic growth and decay and periodic and aperiodic low-amplitude flaring. We show our light curve in Fig. 3 (left panel), while the observer's view of the cloud at ten different times (a simulated VLBI map) is shown in the right-hand panel. The model maser has a light curve, in terms of the brightest specific intensity, that varies by a factor of $\sim 3$ over one rotation. The 

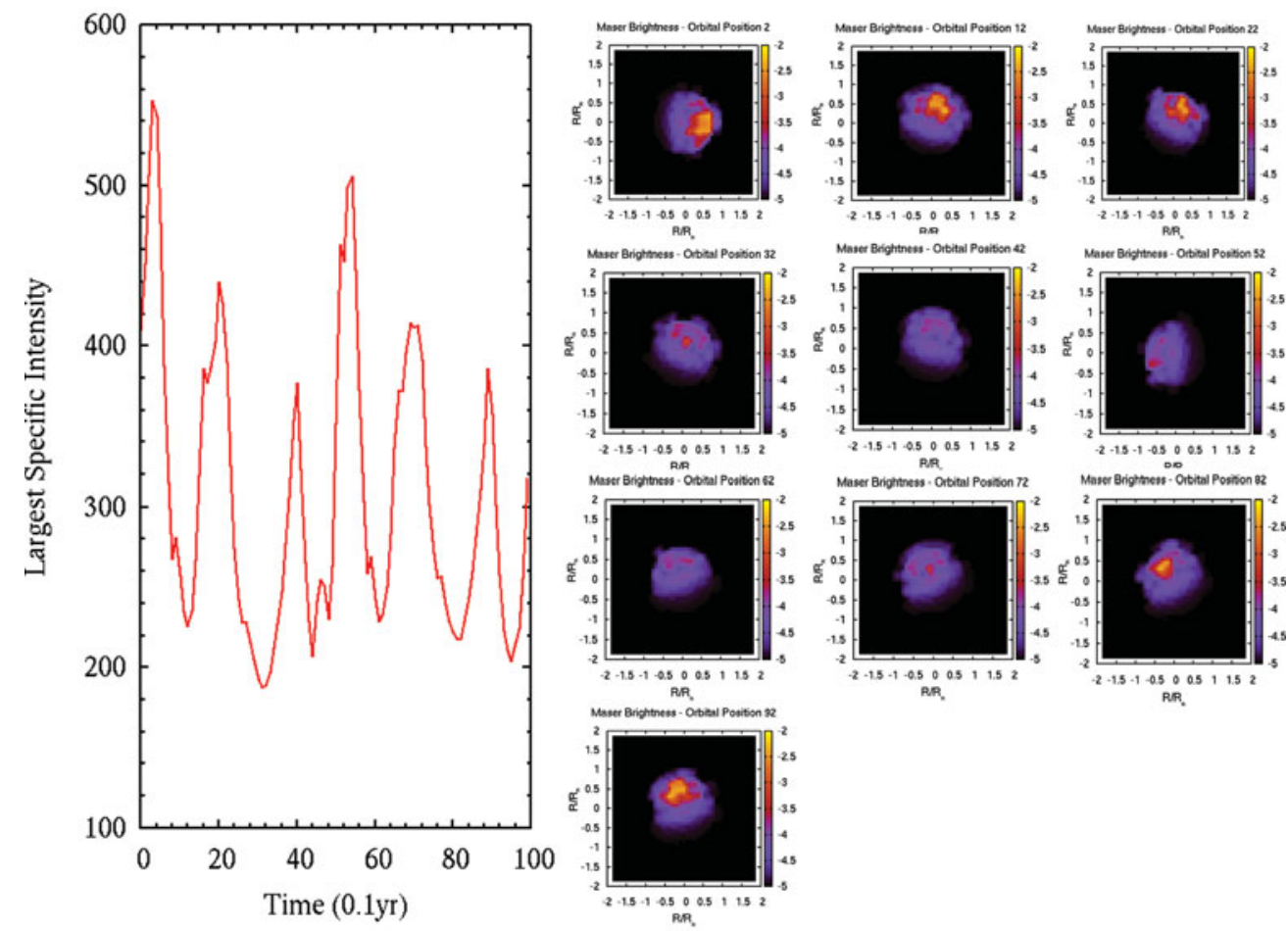

$-2-15-1-0.5000511 .52$
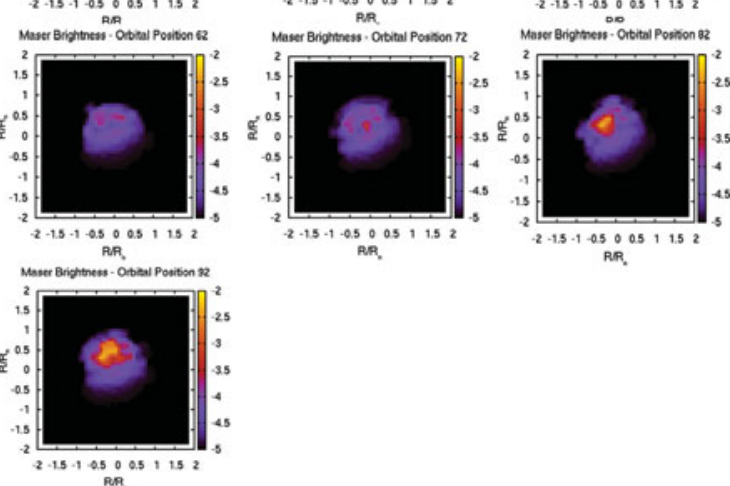

RR.

Figure 3. Left panel: The light curve of the rotating model maser in terms of the peak specific intensity (in multiples of the saturation intensity). Right panel: Snap-shots of the brightness map at various times during the cycle. A lower-depth model has been used so that the whole cloud is easily visible.

simulated map (right-hand panel) shows that the position of brightest emission also varies considerably with time.

\section{References}

Alcock, C. \& Ross, R. R. 1985 ApJ, 290, 433

Elitzur, M. 1992, Astronomical Masers (Dordrecht: Kluwer)

Elitzur, M. \& Asensio Ramos, A. 2006 MNRAS, 365, 779

Goedhart, S., Gaylard, M. J. \& van der Walt, D. J. 2004 MNRAS, 355, 553

Gray, M. D. 2012, Maser Sources in Astrophysics (New York: CUP)

Green, J. A., Gray, M. D., Robishaw, T., Caswell, J. A. \& McClure-Griffiths, N. M. 2014 MNRAS, 440, 2988

Kobayashi, H., Shimoikura, T., Omodaka, T., \& Diamond, P. J. 2000, in: H. Hirabayashi, P. G. Edwards \& D. W. Murphy (eds.), Astrophysical Phenomena Revealed by Space VLBI (Institute of Space and Astronautical Science), p. 109-112

King, J. I.F. \& Florance, E. T. 1964 ApJ, 139, 397

Landi Degl'Innocenti, E. 1988, in: W. Kalkofen (ed.), Numerical Radiative Transfer (Cambridge, CUP), p. 265

Menegozzi, L. N. \& Lamb Jr., W. E. 1978 Phys. Rev. A, 17, 701

Slysh, V. I. 2003, in: J. A. Zensus, M. H. Cohen \& E. Ros (eds.), Radio Astronomy at the Fringe (ASP Conference Series, Vol. 300), p. 239

Strelnitskii, V. S. 1982 Soviet Astron. Lett., 8, 86

Takefuji, K, Imai, H. \& Sekido, M. 2016 PASJ, 68, 86

Zienkiewicz, O. \& Taylor, R. 2000, The Finite Element Method: The basis (Oxford: ButterworthHeinemann) 